\title{
Double-stranded RNA innate immune response activation from long- term adeno-associated virus vector transduction
}

Wenwei Shao, ${ }^{1}$ Lauriel F. Earley, ${ }^{1}$ Zheng Chai, ${ }^{1}$ Xiaojing Chen, ${ }^{1}$ Junjiang Sun, ${ }^{1,2}$ Ting He, ${ }^{1}$ Meng Deng, ${ }^{3}$ Matthew L. Hirsch, ${ }^{1,4}$ Jenny Ting, ${ }^{3}$ R. Jude Samulski, ${ }^{1,5}$ and Chengwen $\mathrm{Li}^{1,6,7}$

${ }^{1}$ Cene Therapy Center, ${ }^{2}$ Division of Pharmacoengineering and Molecular Pharmaceutics, School of Pharmacy, ${ }^{3}$ Lineberger Comprehensive Cancer Center, ${ }^{4}$ Depatment of Ophthalmology, ${ }^{5}$ Department of Pharmacology, ${ }^{6}$ Department of Pediatrics, School of Medicine, and ${ }^{7}$ Carolina Institute for Developmental Disabilities, University of North Carolina at Chapel Hill, Chapel Hill, North Carolina, USA.

Data from clinical trials for hemophilia B using adeno-associated virus (AAV) vectors have demonstrated decreased transgenic coagulation factor IX (hFIX) expression 6-10 weeks after administration of a high vector dose. While it is likely that capsid-specific cytotoxic T lymphocytes eliminate vector-transduced hepatocytes, thereby resulting in decreased $\mathrm{hFIX}$, this observation is not intuitively consistent with restored hFIX levels following prednisone application. Although the innate immune response is immediately activated following AAV vector infection via TLR pathways, no studies exist regarding the role of the innate immune response at later time points after AAV vector transduction. Herein, activation of the innate immune response in cell lines, primary human hepatocytes, and hepatocytes in a human chimeric mouse model was observed at later time points following AAV vector transduction. Mechanistic analysis demonstrated that the double-stranded RNA (dsRNA) sensor MDA5 was necessary for innate immune response activation and that transient knockdown of MDA5, or MAVS, decreased IFN- $\beta$ expression while increasing transgene production in AAV-transduced cells. These results both highlight the role of the dsRNAtriggered innate immune response in therapeutic transgene expression at later time points following AAV transduction and facilitate the execution of effective strategies to block the dsRNA innate immune response in future clinical trials.

Conflict of interest: RJS is the founder of and a shareholder at Asklepios BioPharmaceutical and Bamboo Therapeutics Inc. He holds a patent (9475845) that has been licensed by the University of North Carolina at Chapel Hill to Asklepios BioPharmaceutical, for which he receives royalties. He has consulted for Baxter and has received payment for speaking. MLH has also received royalties from Asklepios BioPharmaceutical related to a patent (9447433).

Submitted: February 13, 2018

Accepted: May 17, 2018

Published: June 21, 2018

\section{Reference information:} JCI Insight. 2018;3(12):e120474. https://doi.org/10.1172/jci. insight.120474.

\section{Introduction}

Adeno-associated virus (AAV) vectors encoding factor IX (FIX) have been successfully applied in clinical trials in patients with hemophilia B (1-4). Following AAV-FIX administration, transgene-derived FIX serum levels, generally, increased over time. However, some patients treated with AAV-FIX demonstrated decreased circulating FIX concomitant with elevated liver enzymes at weeks 6-10 (2-4). Administration of prednisone prevented the FIX decrease and restored FIX to previous levels in the blood $(2,3)$. This phenomenon was never observed in preclinical trials using rodents and large animals (5-13). Since AAV capsid-specific cytotoxic T lymphocytes (CTLs) were detected in these patients $(2,3)$, it has been postulated and demonstrated, in part, that the therapeutic failure results from CTL clearance of AAV-transduced hepatocytes $(14,15)$. However, this clearance model is not fully supported by the following evidence. First, kinetic studies of AAV capsid antigen presentation report that efficient antigen presentation occurs immediately after AAV administration and then gradually decreases to undetectable levels at later time points after transduction $(16,17)$. These data imply that capsid-specific CTLs eliminate most AAV-transduced cells at early time points but would not effect transgene expression at a later times after administration. Second, if CTLs mediated elimination of AAV-transduced target cells, then prednisone application would not restore the circulating FIX to previous levels. Third, in some patients with circulating capsid-specific CTLs there was no inhibition of FIX expression (3). Therefore, mechanisms other than a capsid-specific CTL response may play a role in decreased FIX over time in patients receiving AAV-FIX gene therapy. 
It has been demonstrated that the innate immune response is immediately activated following AAV administration via TLR9 and TLR2 recognition $(18,19)$; however, there are no studies about the role of innate immunity at later time points after AAV administration and no studies regarding its potential role in regulation of transgene expression. AAV is a single-stranded (ss) DNA virus with two major genes involved in replication and capsid production flanked by inverted terminal repeats (ITRs). Replacement of viral genes with a therapeutic cassette (composed of the promoter, therapeutic transgene, and a poly A tail) forms the AAV vector construct. Inherent promoter activity of the AAV ITRs of a single transgenic genome $(20,21)$ implicates that plus strand RNA transcribed from the 5 '-ITR and minus strand RNA from the 3'-ITR could be generated in AAV-transduced cells in a cassette-independent manner. Theoretically, ITR or bidirectional promoter-generated RNA from transgenic AAV cassettes (both polarities are individually packaged) could result in opposite polarity complementary transcripts that trigger the double-stranded RNA-mediated (dsRNA-mediated) innate immune response. This assumption was supported by our findings that transgene expression was increased when a plasmid with the 3 '-ITR deletion was used via transfection analysis (Supplemental Figure 1; supplemental material available online with this article; https://doi.org/10.1172/jci.insight.120474DS1) and insertion of a reversed polyA sequence between $5^{\prime}$-ITR and the start codon dramatically decreased transgene expression (Supplemental Figure 2). The minus strand of RNA transcribed by the 3 '-ITR promoter might serve as antisense RNA to knock down transgene expression. The plus strand RNA and minus strand RNA generated from the AAV ITR promoters on both terminals are able to anneal and form a dsRNA in the cytoplasm of AAV-transduced cells. Additionally, it has been shown that some promoters for gene delivery have bidirectional transcription function to generate minus strand RNA (22), which could also form dsRNA in a circularized vector. Another possibility for the formation of dsRNA from gene delivery is the secondary structure formation of mRNA from the transgene cassette due to modification of transgene cDNA sequences. These dsRNA formation pathways could potentially activate an innate immune response.

It is well known that MDA5 and RIG-I are cytoplasmic viral RNA sensors capable of activating type I interferon signaling pathways after virus infection, so they play a critical role in antiviral innate immunity. MDA5 and RIG-I share high sequence similarity and a common signaling adaptor, mitochondrial antiviral signaling (MAVS), but they play nonredundant functions in antiviral immunity by recognizing different viruses or viral RNA $(23,24)$. RIG-I recognizes $5^{\prime}$-triphosphorylated blunt-ended, short dsRNA or ss RNA hairpins that are often present in a variety of positive- and negative-strand viruses (25-27). MDA5 recognizes relatively long dsRNA in the genome of dsRNA viruses or dsRNA replication intermediates of positive-strand viruses, such as encephalomyocarditis virus and poliovirus (26-29).

Herein, the innate immune response to AAV vector transduction at different time points after administration is investigated to address the critical clinical question regarding the influence of innate immunity on transgene-derived FIX levels in a kinetic manner after AAV vector transduction. We found that innate immunity activation was triggered at both early and late time points after AAV transduction and the late innate immune response activation was mediated by cytosolic dsRNA sensors. Blocking of the cytosolic dsRNA sensor or adaptor blunted late IFN- $\beta$ expression and increased transgene expression in AAV-transduced cells, a finding similar to the response following prednisone application observed in the hemophilia $B$ clinical trial (2). The collective results invoke a transgenic genome-derived dsRNA-induced innate response as an alternative mechanism for therapeutic failure of forthcoming clinical data regarding AAV gene therapy for hemophilia B.

\section{Results}

IFN- $\beta$ inhibits transgene expression in AAV-transduced cells. Type I IFN- $\beta$ expression is the hallmark of innate immune activation. To study the effect of innate immune response activation on transgene expression, we investigated the effect of IFN- $\beta$ on transgene expression after AAV transduction in vitro. HeLa cells were infected with AAV2/luciferase vector encoding a firefly luciferase transgene; 24 hours later, IFN- $\beta$ at different doses was added and luciferase activity was measured at several time points thereafter. The results demonstrate an inverse relationship between the administered concentration of IFN- $\beta$ and transgene-derived luciferase activity (Figure 1A). When IFN- $\beta$ was added every day from day 1 after AAV transduction, much stronger inhibition of luciferase expression was observed from a long-term culture (Figure 1B). This result suggests that innate immune response activation may inhibit some aspect of productive AAV vector transduction. 
A

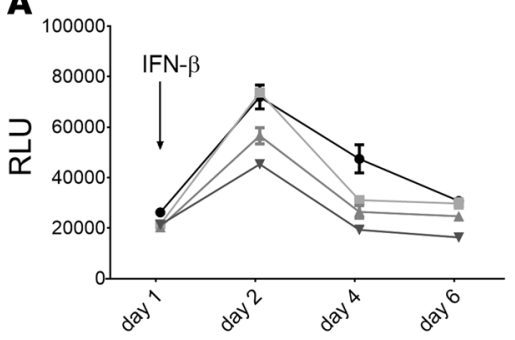

B

IFN- $\beta$

- $0 \mathrm{ng} / \mathrm{mL}$

$0.005 \mathrm{ng} / \mathrm{mL}$

$\pm 0.05 \mathrm{ng} / \mathrm{mL}$

$=0.5 \mathrm{ng} / \mathrm{mL}$

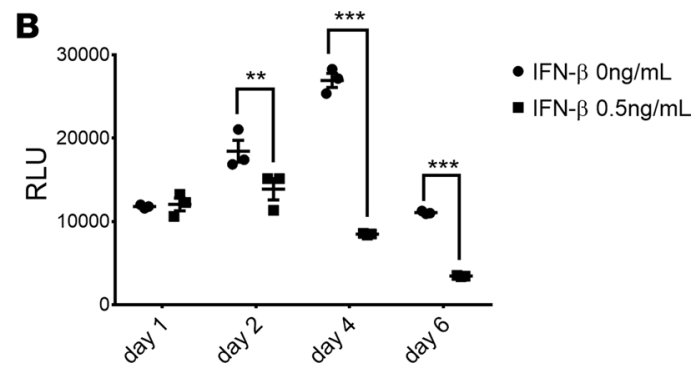

Figure 1. IFN- $\beta$ inhibited AAV transgene expression in the HeLa cell line. HeLa cells were transduced with $5 \times 10^{3}$ particles of AAV2/luciferase per cell. (A) After 24 hours, recombinant human IFN- $\beta$ was added to the medium at a different dose. Transgene expression was detected by luciferase assay at days 1, 2, 4, and 6 after supplementation of IFN- $\beta$. (B) Recombinant human IFN- $\beta$ was added to the medium every day at 0.5 ng/ml. Transgene expression was detected by luciferase assay at days $1,2,4$, and 6 . ${ }^{* *} P<0.001$, when compared with no IFN- $\beta$ treatment, as measured by 2 -way ANOVA. Results are representative of 3 independent experiments.

Poly (I:C) inhibits AAV transduction. In clinical trials for hemophilia, some patients showed decreased circulating FIX at weeks 6-10 after AAV-FIX administration (2-4). At this later time point after administration, the possibility of the innate immune response being triggered by AAV vectors from pattern recognition receptors (PRRs) in endosomes or sensors for DNA in cytoplasm seems low, as the persistent form of AAV is likely double-stranded exosomes. We presume that dsRNA could be generated from AAV vector-mediated transgene delivery and could activate the innate immune response. To determine whether innate immunity from dsRNA effects vector-derived transgene activity, cells lines were transfected with polyinosinic-polycytidylic acid [poly (I:C)], a synthetic analog of dsRNA, at different time points relative to AAV/luciferase vector administration. Regardless of the time points, transfection of poly (I:C) inhibited luciferase activity in both HeLa and Huh7 cells (Figure 2). This result indicates that innate immune activation mediated by poly (I:C), as a synthetic analog of dsRNA, is able to inhibit transgene expression. The data indicate that the innate immune response triggered from dsRNA effects AAV transduction.

Minus strand RNA generation from AAV 3'-ITR after AAV transduction. To investigate whether minus strand RNA could be generated from the AAV 3'-ITR promoter, HeLa cells were infected with AAV2/luciferase vectors and harvested 8 days later for RNA extraction. The cDNA synthesis was performed using sense or antisense primers of the luciferase transgene. Two pairs of luciferase-specific PCR primers were used to detect plus or minus strand transcripts (Figure 3A). Both plus and minus strand transcripts were detected after AAV transduction, and there was no PCR product when the RNA was used as a template (Figure 3B). The minus strand transcripts were only detected when the cDNA template was diluted 200-fold. However, even at a 2,000-fold dilution of the cDNA template, we were still able to detect the plus strand transcript (Figure $3 \mathrm{~B}$ ). This result indicates that the transcripts in a reverse orientation can be generated from AAV transduction and that the efficiency of minus strand RNA formation is much lower than that of plus strand RNA. It also supports the possibility that a plus strand RNA and a minus strand RNA that is generated from a different orientation are able to form dsRNA in AAV-transduced cells.

dsRNA innate immune response is triggered from late AAV transduction in HeLa cells. To study whether the transduction of AAV activates the dsRNA innate immune response, we examined the expression kinetics of the dsRNA sensors MDA5 and RIG-I at the transcriptional level. As shown in Figure 4A, the upregulation of MDA5 was observed at days 6 and 8 after self-complementary AAV/GFP (scAAV/GFP) vector administration to HeLa cells. RIG-I expression was not significantly altered during AAV transduction in HeLa cells (Figure 4B). The IFN- $\beta$ mRNA was significantly higher starting at day 8 after vector administration (Figure 4C). The upregulation of MDA5 and IFN- $\beta$ expression was also detected at an early time point after AAV infection. As a control, the expression of MDA5, RIG-I, and IFN- $\beta$ were increased in HeLa cells at 8 hours after the poly (I:C) transfection and decreased to the baseline at day 3 (Supplemental Figure 3). In addition, the transgene expression was measured at different time points after AAV transduction (Figure 4D). The expression of the GFP transgene reached a peak at day 4 and then decreased at day 6 . We also examined MDA5 by Western blotting, which demonstrated an increased abundance in the AAV vectortreated cells at day 8 after AAV transduction (Figure 4E). These results suggest that the AAV transduction activates a dsRNA-mediated innate immune response at 6 days after infection. 
HeLa

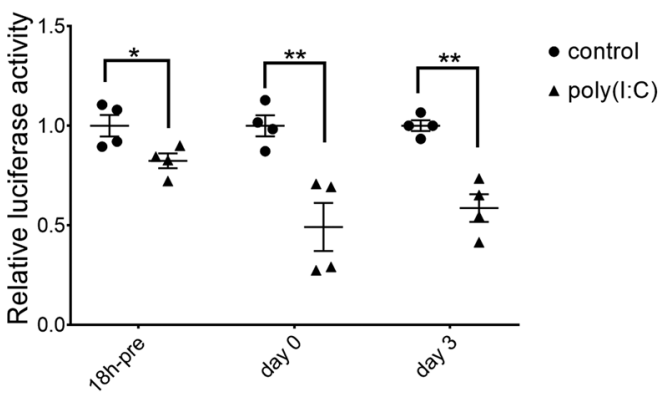

Huh7

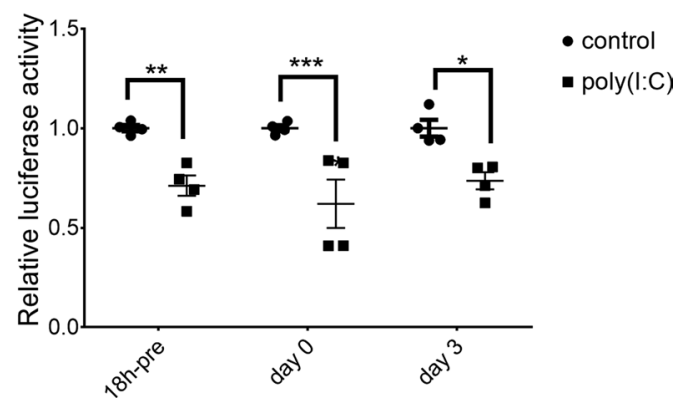

Figure 2. Poly (I:C) inhibited AAV transgene expression in cell lines. HeLa or Huh7 cells were transduced with $5 \times 10^{3}$ particles of AAV2/luciferase per cell. $2 \mu \mathrm{g} / \mathrm{ml}$ poly (I:C) was added at different time points: 18 hours before AAV transduction, day 0 , or day 3. Luciferase expression was detected 3 days after poly (I:C) transfection. ${ }^{*} P<0.05,{ }^{* *} P<0.01,{ }^{* *} P<0.001$, when compared with control group, as measured by 2 -way ANOVA. Results are representative of 4 independent experiments.

$A A V$ transduction-mediated dsRNA innate immune response activation is cell specific and transgene and dose dependent. In hemophilia B clinical trials, transgene FIX was driven by a liver-specific promoter in an effort to restrict expression to hepatocytes (2-4). The results from the above studies have demonstrated that the dsRNA immune response is triggered at a later time after AAV transduction in HeLa cells, a nonhepatocyte cell line (Figure 4). Next, we wondered whether the dsRNA innate immune response was also triggered in other human cell lines, including cell lines that were derived from hepatocytes. After infection of AAV2/ GFP vectors, at different time points, dsRNA response was evaluated. The upregulation of MDA5 was only observed in the human hepatocyte Huh7 and HepG2 cells and not in the HEK293 cells. The IFN- $\beta$ expression was increased in all 3 of the cell lines (Figure 5A).

To study the activation of the dsRNA-mediated innate immune response from different transgenes, we transduced HeLa cells with AAV2 encoding different transgenes, including luciferase, shRNA scramble, and antitrypsin. RNA was harvested for analysis at day 8 after AAV transduction. Compared with the control group, higher expression of MDA5 was observed in HeLa cells transduced with ssAAV/ GFP, AAV2/luciferase, and AAV2/shRNA scramble but not with AAV2/AAT (Figure 5B). However, the IFN- $\beta$ expression was upregulated in all AAV2 vector-transduced cells, regardless of the transgenes.

We also tested the dsRNA-mediated innate immune response to different doses of AAV virus. HeLa cells were transduced with scAAV2/GFP with doses of 100, 5,000, and 25,000 virus particles per cell. The upregulation of MDA5 and IFN- $\beta$ expression at day 8 after AAV transduction was dose dependent (Figure 5C).

A

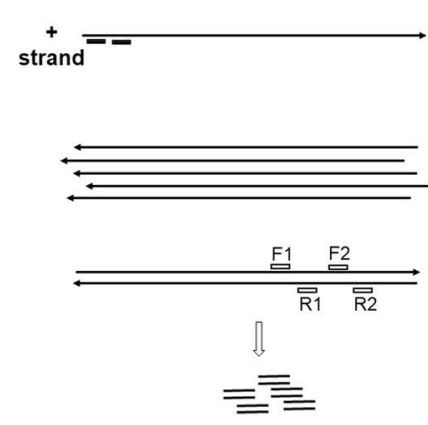

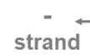

Reverse transcription

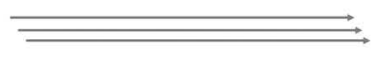

PCR

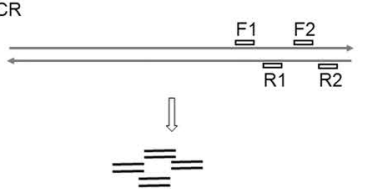

B
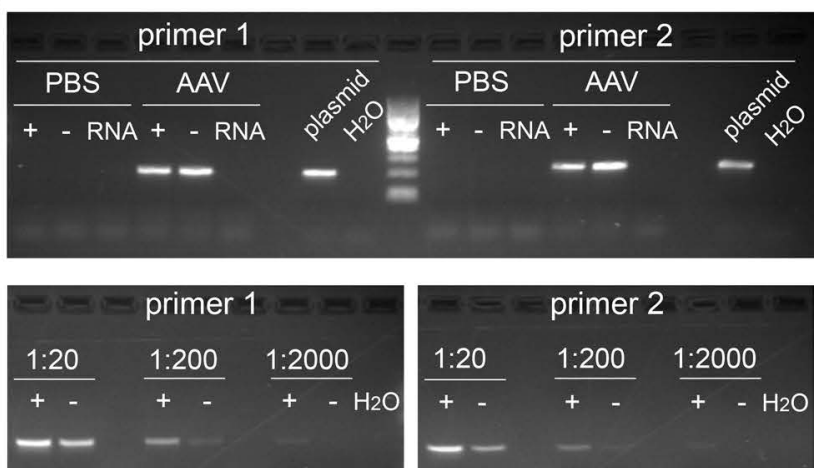

Figure 3. Minus strand transcript generation in AAV-transduced cells. (A) Overview of the gene-specific reverse transcription to detect either plus or minus strand transcripts. HeLa cells were harvested at day 8 after AAV2/luciferase transduction. The RNA was extracted and treated with DNase. Specific primers for plus strand or minus strand luciferase were used to synthesize different orientations of the cDNA. PCR was performed to detect the transcripts in different orientations of cDNA using primer pair 1 (F1 and R1) and primer pair 2 (F2 and R2). (B) PCR products are shown. PBS was used as a negative control with no AAV virus. The pTR/luciferase plasmid served as positive control for the PCR. RNA was used as a template to eliminate the possibility of AAV genome DNA contamination in extracted RNA. To measure the yield of transcripts, cDNA in different orientations was diluted to 20-, 200-, or 2,000-fold as PCR templates. 
$\mathbf{A}$

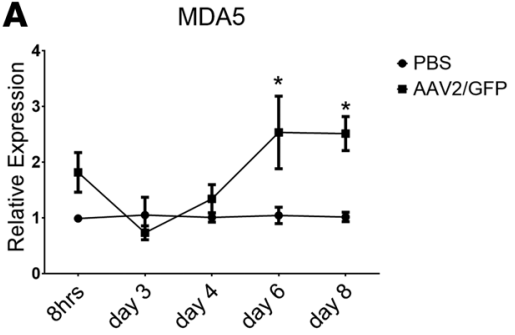

D

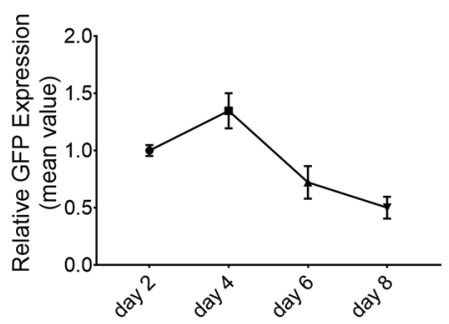

RIG-I

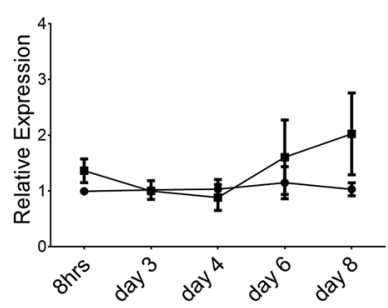

E

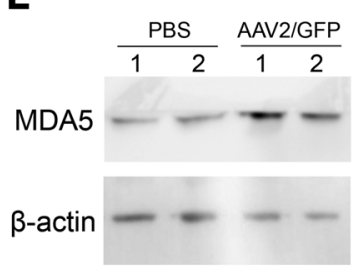

C

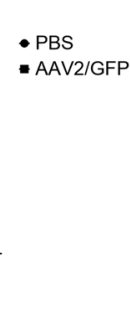

IFN- $\beta$

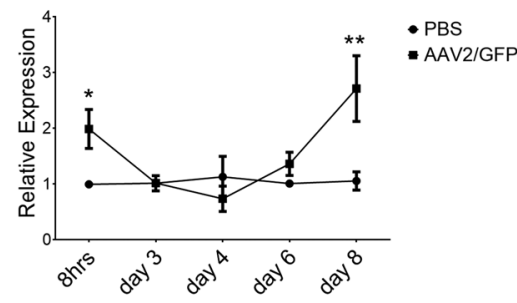

MDA5

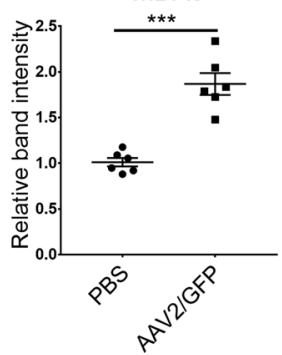

Figure 4. dsRNA immune response is activated at a later time point after AAV transduction. HeLa cells were transduced with $5 \times 10^{3}$ particles of scAAV2/ GFP per cell. The expression of MDA5 (A), RIG-I (B), and IFN- $\beta$ (C) in HeLa cells was detected by Q-PCR at different time points after transduction. ${ }^{*} P<$ $0.05,{ }^{* *} P<0.01$, when compared with the PBS group, as measured by 2 -way ANOVA. The data represent the mean \pm SEM from 3 independent experiments. For each experiment, the PBS- or AAV-infected group contained 2 or 3 wells of cells. For Q-PCR data analysis, one sample from the PBS group was normalized to one in each time point of each experiment. (D) GFP expression level in HeLa cells was detected by flow cytometry at different time points after AAV2/GFP transduction. (E) MDA5 expression in HeLa cells in each group was detected by Western blot 8 days after scAAV2/GFP transduction. The relative level of MDA5 expression was calculated based on the intensity of $\beta$-actin protein from 3 independent experiments. ${ }^{* * *} P<0.001$, when compared with the PBS group, as measured by 2-tailed Student's $t$ test.

The dsRNA innate immune response is induced in AAV/GFP-transduced primary human hepatocytes. Next, activation of the dsRNA innate immune response was investigated in the human primary hepatocytes following AAV vector transduction. scAAV2/GFP vectors were used to transduce primary human hepatocytes from 12 different subjects ex vivo. At different time points after transduction, RNA was harvested for cDNA analysis of MDA5, RIG-I, and IFN- $\beta$. MDA5 was upregulated in 6 of 12 subjects following AAV administration, with an apparent peak at day 5 , or later, followed by a decline to the baseline thereafter (Figure 6A). A higher expression of RIG-I was only observed in 3 subjects (subjects 1, 5, and 12) (Figure 6A). Another 6 subjects did not show any change in the expression levels of MDA5 or RIG-I (Figure 6B). However, a higher expression of IFN- $\beta$ was detected at later time points in all subjects after AAV transduction (Figure 6). There was no obvious pattern for high expression of IFN- $\beta$, and, in most cases, the increased IFN- $\beta$ expression was accompanied by MDA5 expression at a late time point ( $\geq 5$ days) after AAV transduction. In some subjects, high IFN- $\beta$ expression was detected at very early time points (within 1 day) after AAV transductions (subjects 1, 3, 7, and 9), but there was no change for the dsRNA sensors. These results perhaps support the innate immune response activation via the dsDNA/TLR9 pathway, as reported in other studies $(18,19,30)$.

dsRNA innate immune response induction in AAV/FIX-opt-transduced primary human hepatocytes. Next, we tested whether AAV vectors packaged with a therapeutic FIX cassette also triggered the dsRNA innate immune response $(4,6)$. Human primary hepatocytes from 10 subjects were transduced with AAV vectors harboring the clinically applied FIX cassette: optimized human FIX with a R338L mutation for enhanced coagulation activity (hFIX-R338L-opt) (6). After transduction with AAV2/hFIX-R338L-opt, MDA5 and IFN- $\beta$ were upregulated in 5 of 10 subjects at day 5 or later (Figure 7A) and the other 5 subjects just showed only a high expression of IFN- $\beta$ (Figure 7B). RIG-I was upregulated in subjects 8 and 12 at day 7 after AAV transduction (Figure 7A). This result indicates that the dsRNA innate immune response is activated in human primary hepatocytes transduced with AAV vectors encoding a clinically therapeutic transgene (Figure 7).

$A A V$ transduction induced activation of the dsRNA innate immune response in humanized mouse livers. The above results support that the cytosolic dsRNA innate immune response in human cells is activated at a later time following AAV transduction in vitro. Next, we examined the dsRNA innate immune response in human 
A

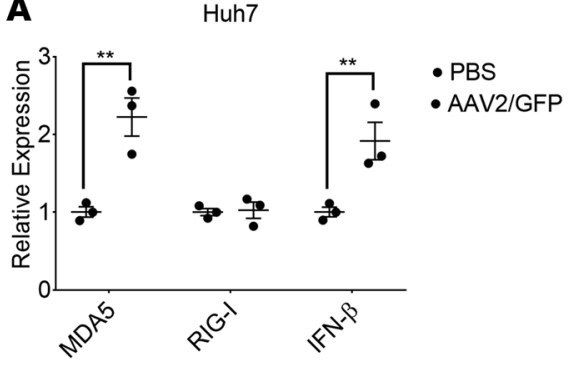

B

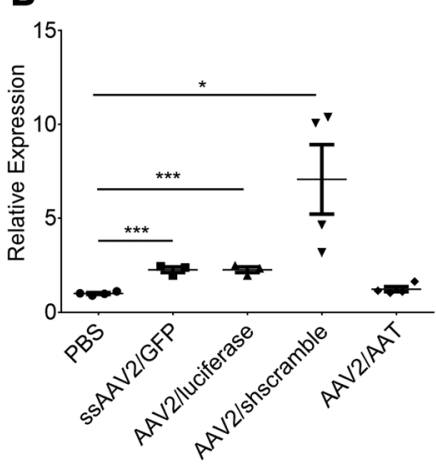

HepG2

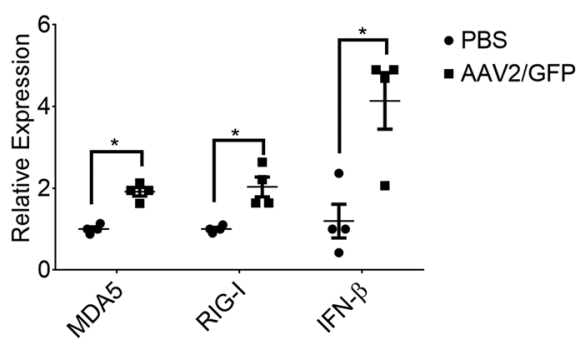

RIG-I

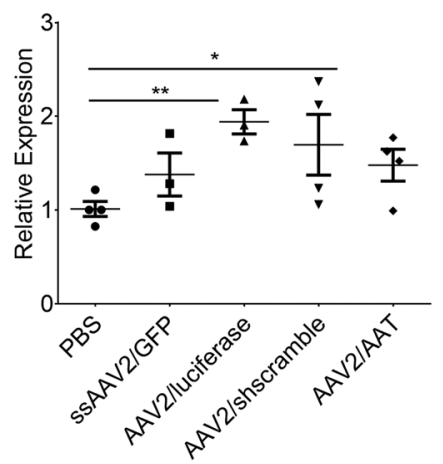

HEK293

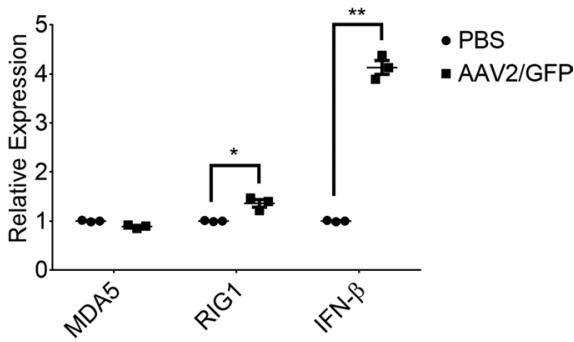

IFN- $\beta$

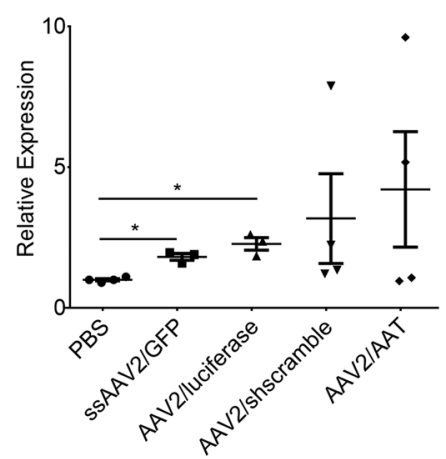

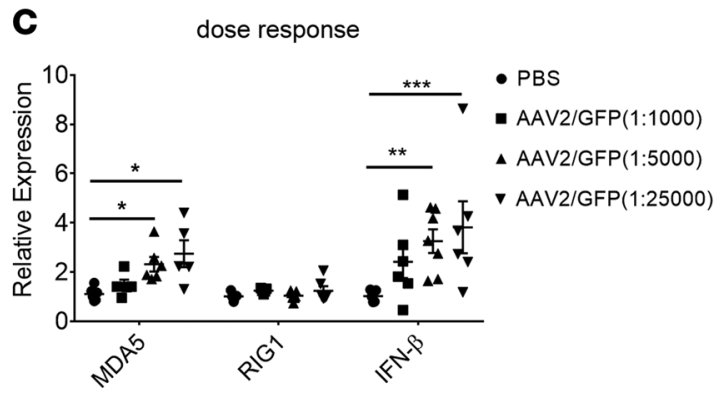

Figure 5. dsRNA response profile in different cell lines and different transgenes. (A) Huh7, HEK293, and HepG2 cells were transduced with $5 \times 10^{3}$ particles of AAV2/GFP per cell. The expression of MDA5, RIG-I, and IFN- $\beta$ was detected by Q-PCR at day $7 .{ }^{*} P<0.05,{ }^{* *} P<0.01$, when compared with the PBS group, as measured by 2-way ANOVA. The data present the mean \pm SEM from 3 independent experiments. (B) HeLa cells were transduced with AAV2 containing different transgenes at $5 \times 10^{3}$ particles per cell. The expression of MDA5, RIG-I, and IFN- $\beta$ was detected by Q-PCR at day 7 after AAV transduction. For Q-PCR data analysis, samples from the PBS group were normalized to 1 in each experiment. ${ }^{*} P<0.05$, ${ }^{* *} P<0.01$, ${ }^{* *} P<0.001$, when compared with the PBS group, as measured by 2-tailed Student's $t$ test. (C) HeLa cells were transduced with different doses of scAAV/GFP $(1,000,5,000$, or 250,000 particles of AAV2/GFP per cell). The expression of MDA5, RIG-I, and IFN- $\beta$ was detected by Q-PCR at day 7. ${ }^{*} P<0.05,{ }^{* *} P<0.01,{ }^{* * *} P<0.001$, when compared with the PBS group, as measured by 2 -way ANOVA. The data present the mean \pm SEM from 3 independent experiments, and each group contained 2 or 3 wells of cells for each experiments.

hepatocytes in vivo using a human chimeric mouse model. In these mice, human hepatocytes were engrafted into mouse liver with a 70\% repopulation. In the first set of experiments, we injected clinical vectors AAV8/ hFIX-opt in 2 mice, and, at week 8, mouse liver was harvested for expression analysis. Both MDA5 and RIG-I cDNA were increased in the human hepatocytes of mice treated with AAV vectors, and MDA5 upregulation was confirmed by Western blot (Figure 8A). In addition, IFN- $\beta$ expression was higher in the AAV8/hFIXopt-treated mice than that in no treatment controls (Figure 8A). In the second experiment, liver was harvested at weeks 4 or 8 following AAV8/hFIX-opt systemic injection. The mRNA level for MDA5 had increased at week 4 and had decreased to the same level as that in the controls 8 weeks after AAV vector injection (Figure 8B). A higher expression of MDA5 in the second experiment was also confirmed at the protein level (Figure $8 \mathrm{C}$ ). The AAV-treated mice had a high IFN- $\beta$ expression not only at week 4, but also at week 8 (Figure 8B).

Blockage of the dsRNA activation pathway increases transgene expression and inhibits IFN- $\beta$ expression from $A A V$-transduced cells. In the experiments described above, the induction of the innate immune response and/ or the addition of IFN- $\beta$ decreased AAV transduction (Figure 1). Next, we investigated whether blocking the dsRNA innate immune response effects the transgene expression after AAV transduction. Since MDA5 
A

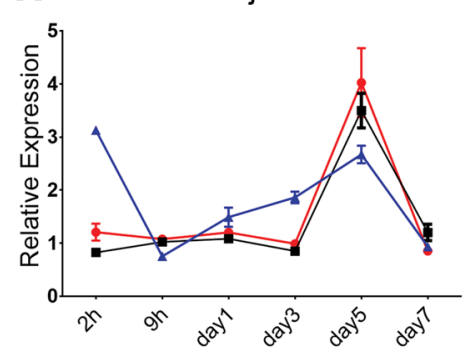

subject 7

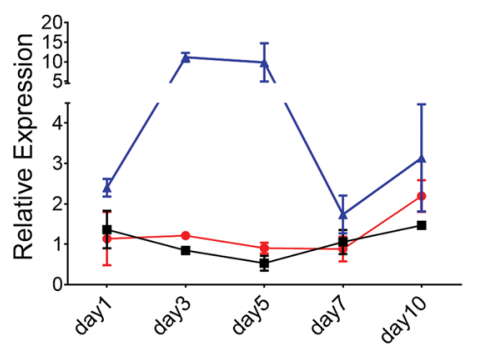

B

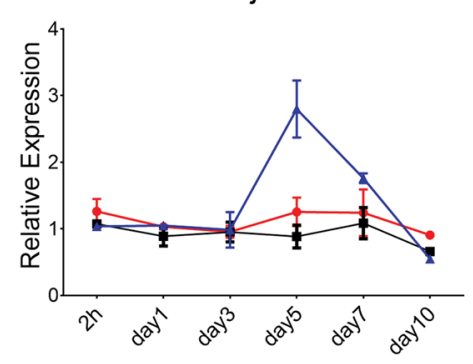

subject 10

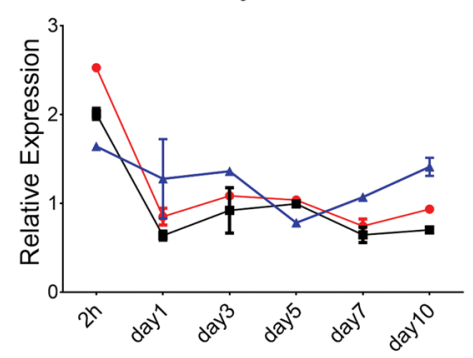

subject 3
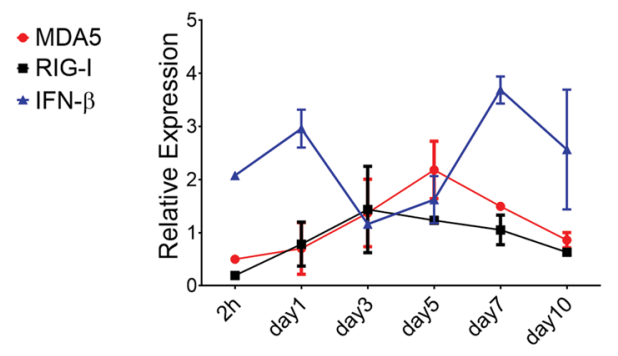

subject 4

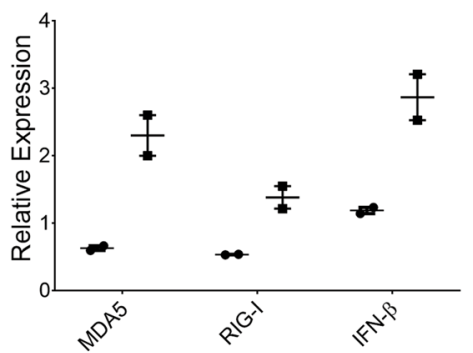

subject 6

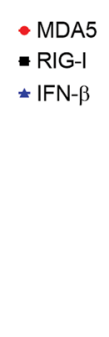

subject 8
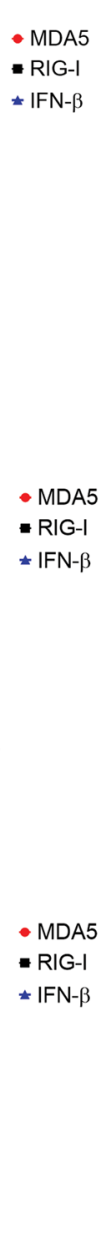
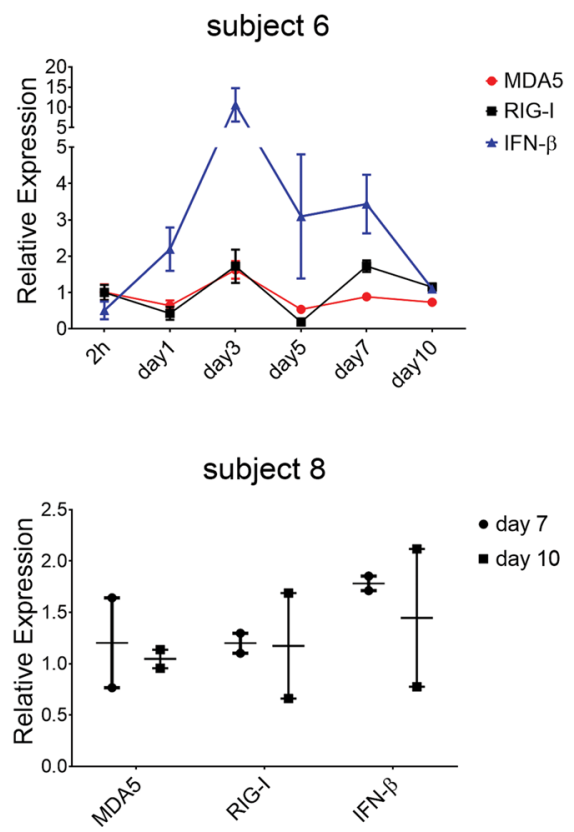

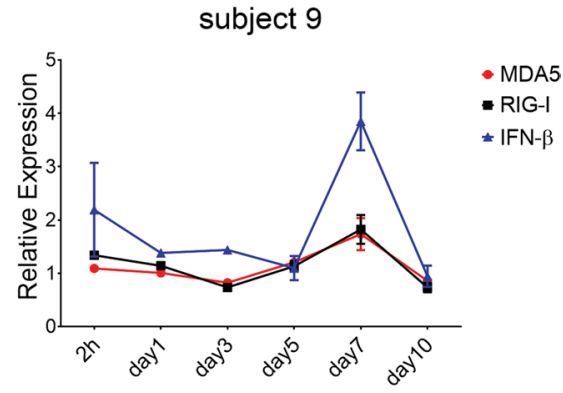

subject 11

subject 5

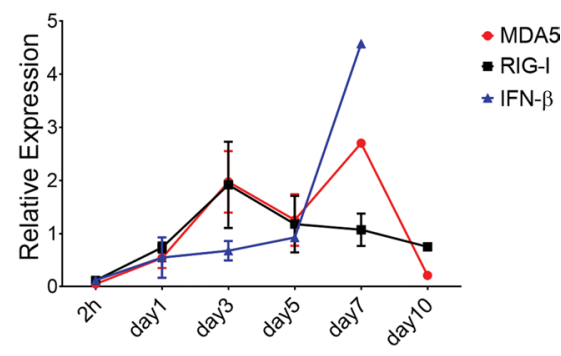

subject 12

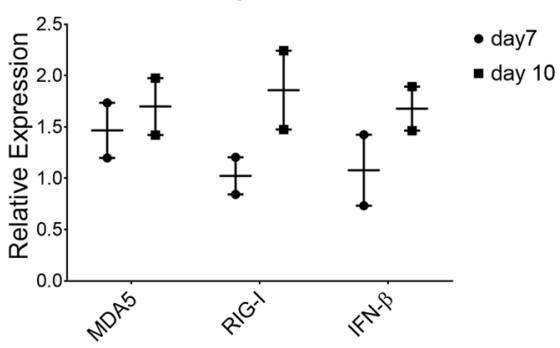

subject 9

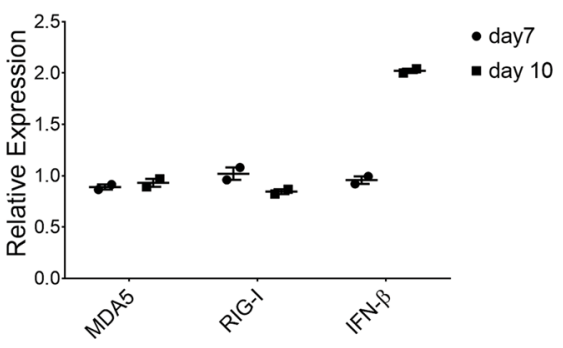

Figure 6. dsRNA innate immune response in human primary hepatocytes after scAAV2/GFP transduction. Fresh human primary hepatocytes from 12 individuals were transduced by scAAV2/GFP with $5 \times 10^{3}$ particles per cell. The expression of MDA5, RIG-I, and IFN- $\beta$ was detected by Q-PCR at different time points after AAV transduction. For relative gene expression calculation, the gene expression of the PBS group at each time point was normalized to 1 , which is not shown in the graph. Biological duplicates were used in each sample. (A) MDA5 expression was $\geq 2$-fold upregulated following AAV administration at day 5 or later. (B) MDA5 expression was similar to PBS following AAV administration at day 5 or later.

is a major dsRNA sensor in HeLa cells following AAV transduction (Figure 4), siRNAs specific to MDA5 and MAVS, a common adaptor for MDA5 and RIG-I, were employed to knockdown their expression. The transfection of siRNA was able to efficiently decrease the cellular abundance of MDA5 and MAVS (Figure 9A). At first, we examined the effect of siRNA on the inhibition of poly (I:C) on AAV transgene expression. At day 3 after AAV2/luciferase transduction, poly (I:C) was added. At day 4 siRNA was transfected. At 48 or 72 hours after siRNA, the transgene expression was measured. The luciferase activity was significantly increased at both 48 and 72 hours when siRNA for MDA5 or MAVS was used (Figure 9B). Next, at day 4 after AAV transduction, siRNA was transfected and IFN- $\beta$ expression was measured 48 and 
A subject 5

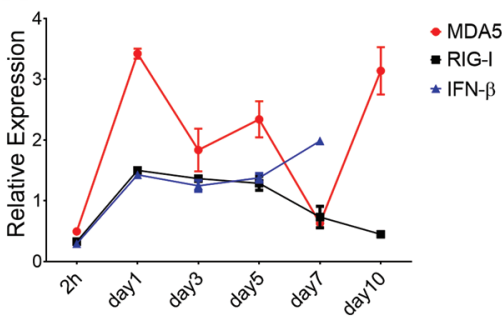

subject 4

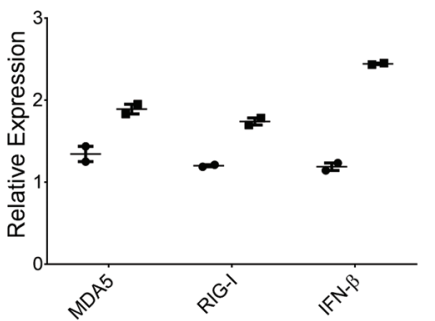

B

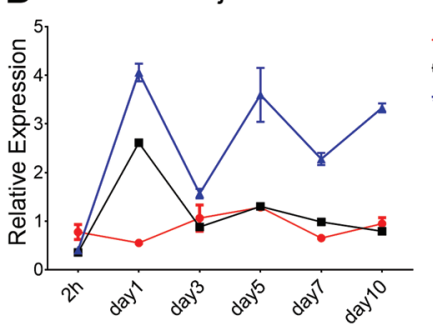

subject 10

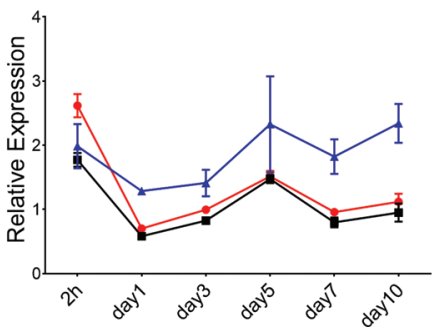

subject 7

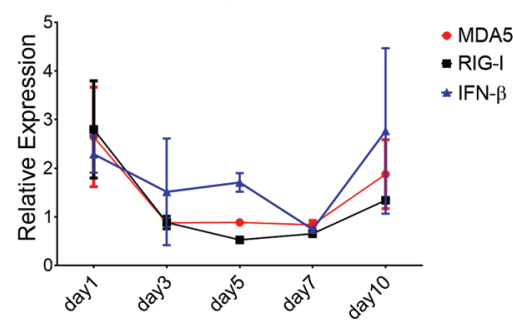

subject 8

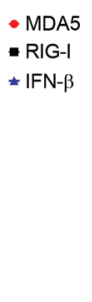

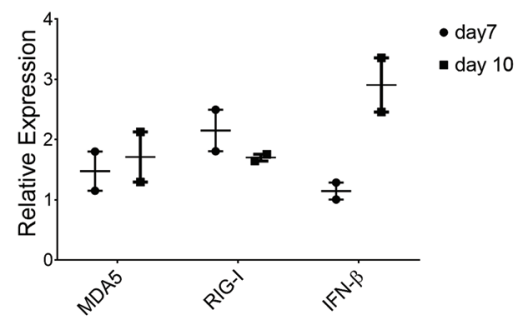

subject 6
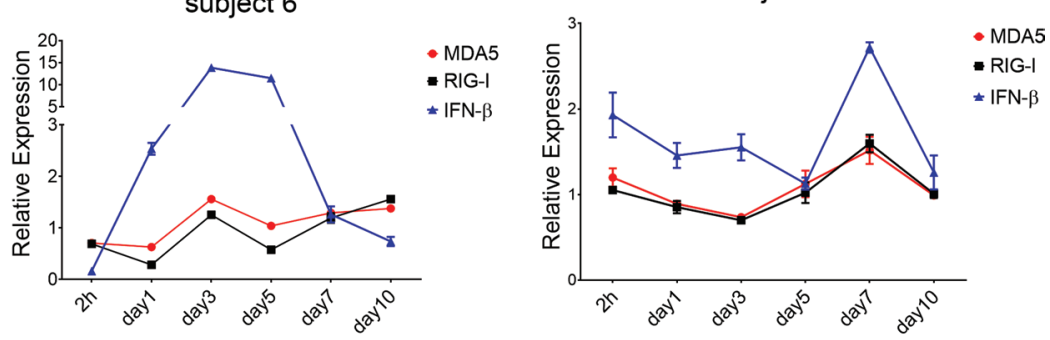

subject 12

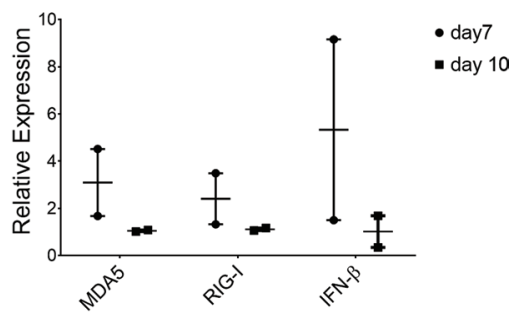

subject 9

subject 11
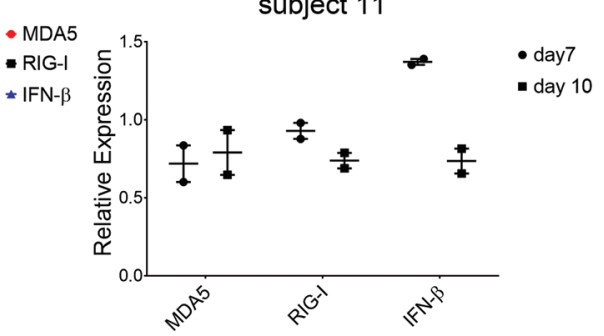

Figure 7. dsRNA innate immune response in human primary hepatocytes after scAAV2/hFIX-opt transduction. Fresh human primary hepatocytes from 10 individuals were transduced by scAAV2/hFIX-opt with $5 \times 10^{3}$ particles per cell. The expression of MDA5, RIG-I, and IFN- $\beta$ was detected by Q-PCR at different time points after AAV transduction. For relative gene expression calculation, the gene expression of the PBS group at each time point was normalized to 1, which is not shown in the graph. Biological duplicates were used in each sample. (A) MDA5 expression was $\geq 2$-fold upregulated following AAV administration at day 5 or later. (B) MDA5 expression was similar to PBS following AAV administration at day 5 or later.

72 hours later. Similar to the finding from poly (I:C) application, higher luciferase activity was achieved following siRNA administration (Figure 9B). In addition, the siRNAs did not activate TLR3 or TLR9 at the baseline (Supplemental Figure 4). Finally, we studied the effect of siRNAs on IFN- $\beta$ expression after AAV transduction. Consistent with the above studies, a high IFN- $\beta$ expression was shown with the siControl RNA transfection (Figure 9C). As expected, the IFN- $\beta$ expression was almost completely inhibited when siRNAs for either MDA5 or MAVS were used at both the RNA and protein levels (Figure 9, C and $\mathrm{D})$. It is interesting to note that the MDA5 upregulation was rescued when siMAVS were used (Figure 9E). Moreover, IFN- $\beta$ can inhibit the increased transgene expression caused by siRNA treatment (Supplemental Figure 5). The siRIG-I was also used to suppress the dsRNA pathway. As a result, the siRIG-I had no effect on the transgene expression, which is consistent with above finding that the RIG-I level did not increase after a long-term AAV transduction (Supplemental Figure 6). These results indicate that the blockage of the dsRNA activation pathway is able to blunt the innate immune response at a late phase following AAV transduction, which leads to a higher transgene expression. 
A

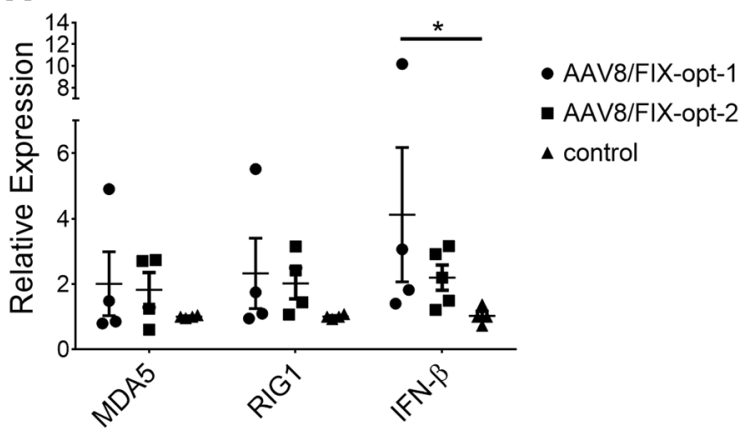

B

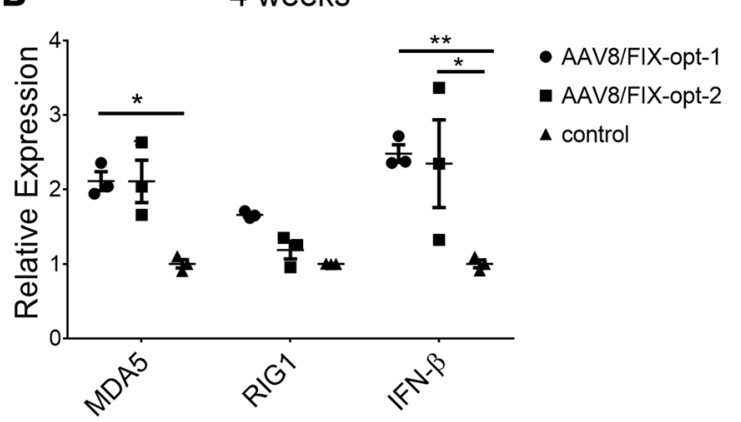

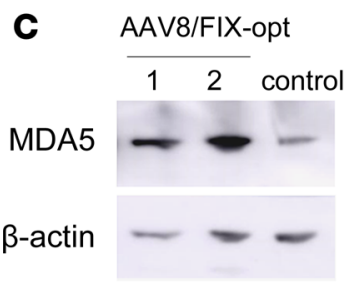

4 weeks
AAV8/FIX-opt

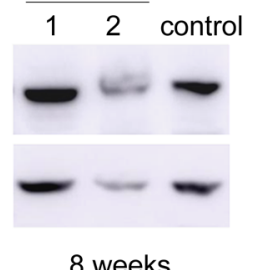

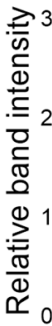

8 weeks
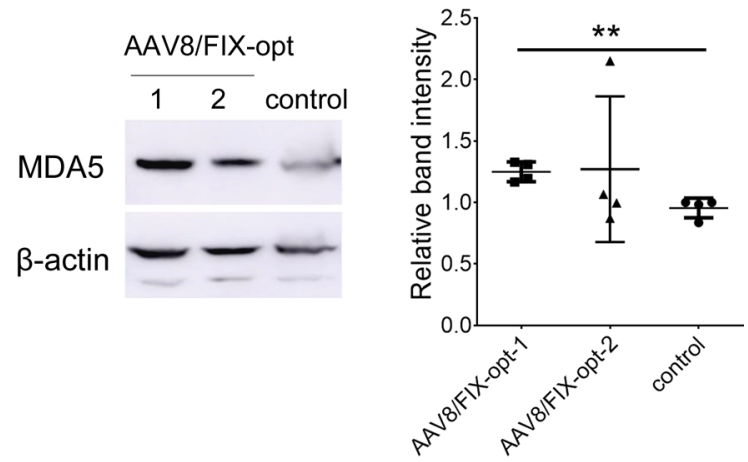

8 weeks

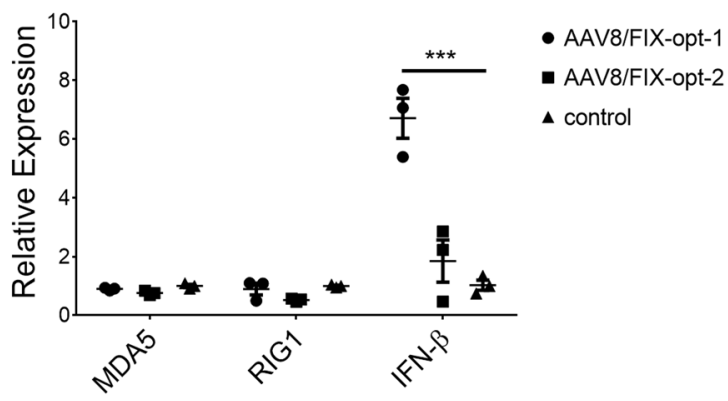

- AAV8/FIX-opt-1

- AAV8/FIX-opt-2

$\triangle$ control

Figure 8. dsRNA response in human hepatocytes from xenografted mice after scAAV8/hFIX-opt transduction. (A) Two xenografted mice with human hepatocytes were injected with $3 \times 10^{11}$ particles of scAAV8/hFIX-opt. The expression of MDA5, RIG-I, and IFN- $\beta$ in human hepatocytes in mice was detected by Q-PCR at 8 weeks after AAV transduction. Three to four pieces of xenografted liver were chosen randomly for RNA extraction. MDA5 protein in the liver was detected by Western blot after 8 weeks. The band intensity was measured to show the relative MDA5 expression based on $\beta$-actin; the data were from 4 separate experiments. ${ }^{*} P<0.05,{ }^{* *} P<0.01$, when compared with the control group; data for multiple comparisons were compared using 2 -way ANOVA, and data for single comparisons were evaluated by the 2-tailed Student's $t$ test. (B) Two xenografted mice with human hepatocytes from another donor were injected with a dose of scAAV8/hFIX-opt. The expression of MDA5, RIG-I, and IFN- $\beta$ in human hepatocytes in mice was detected by Q-PCR at 4 and 8 weeks after AAV transduction. ${ }^{*} P<0.05$, ${ }^{* *} P<0.01$, ${ }^{* *} P<0.001$, when compared with the control group, as measured by 2 -way ANOVA. (C) MDA5 protein in livers from the second experiment was detected by Western blot, and the relative expression level of MDA5 was calculated based on $\beta$-actin intensity; the data were from 4 separate experiments. ${ }^{*} P<0.05$, when compared with the control group, as measured by 2 -way ANOVA.

\section{Discussion}

The innate immune response system is the first line of defense against pathogens, and its activation following AAV (WT or vector) transduction has been noted at earlier time points shortly after infection $(18,19,30$, 31). In this study, we demonstrated for the first time to our knowledge that the innate immune response was triggered at a later time point after long-term AAV transduction. The late innate immune response activation by AAV vectors occurred in different cell types, including primary human hepatocytes. Most importantly, the late innate immune response was also detected in human hepatocytes from the livers of human xenografted mice after AAV transduction. The late innate immune response was mediated via the dsRNA activation pathway, as blocking the dsRNA sensor or adaptor was able to blunt the response and increase AAV transduction. 

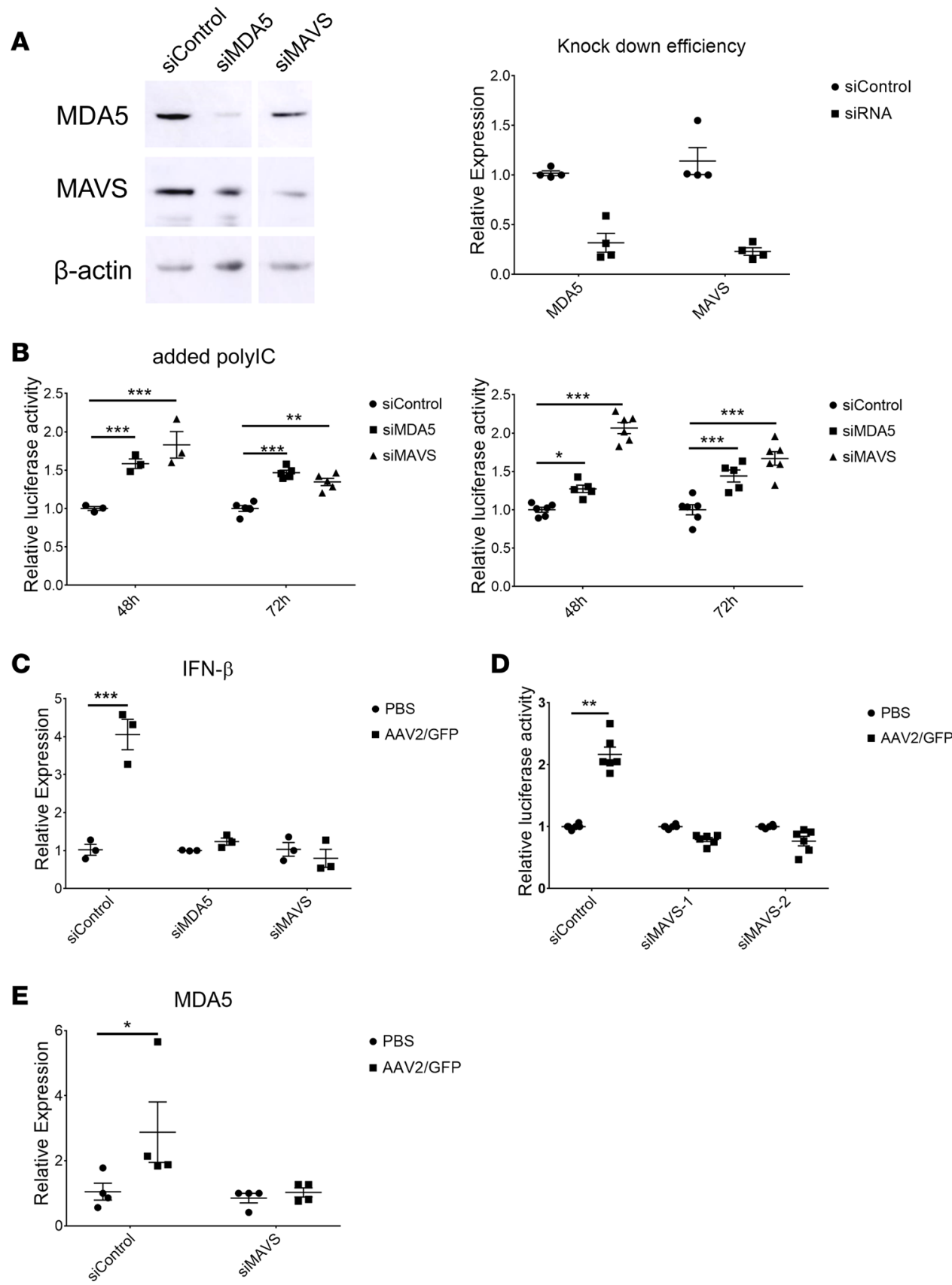

- PBS

- AAV2/GFP

Figure 9. Knock out of dsRNA activation pathway increased AAV transgene expression. (A) HeLa cells were transfected with siControl, siMDA5, or siMAVS. The knockdown efficiency was detected by Western blot and Q-PCR. (B) At day 0, HeLa cells were transduced with $5 \times 10^{3}$ particles of AAV2/luciferase per cell. siRNA was transfected into HeLa cells at day 4, and luciferase expression was detected 48 or 72 hours later. As a control, 2 $\mu \mathrm{g} / \mathrm{ml}$ poly (I:C) was added at day 3 and siRNA was transfected to HeLa cells at day $4 .{ }^{*} P<0.05,{ }^{*} P<0.01,{ }^{* * *} P<0.001$, when compared with the PBS group, as measured by 2-way ANOVA. The data present the mean \pm SEM from 4 independent experiments. (C) After 4 days of AAV transduction, siRNA was transfected to HeLa cells, and IFN- $\beta$ expression was detected by Q-PCR at 48 hours after siRNA transfection. ${ }^{* * *} P<0.001$, when compared with the PBS group, as measured by 2-way ANOVA. The data present the mean \pm SEM from 3 independent experiments. (D) After 4 days of AAV transduction, siRNA and IFN- $\beta$ promoter reporter plasmids were cotransfected to HeLa cells and then luciferase activity was measured after 72 hours. ${ }^{*} P<0.01$, when compared with the PBS group, as measured by 2 -way ANOVA. (E) After 4 days of AAV transduction, siRNA was transfected to HeLa cells, and MDA5 expression was detected by Q-PCR at 48 hours after siRNA transfection. ${ }^{*} P<0.05$, when compared with the PBS group, as measured by 2 -way ANOVA. The data present the mean \pm SEM from 4 independent experiments. 
For innate immune response activation, generally recognition of pathogen-associated molecular patterns by PRRs upregulates costimulatory molecules and inflammatory cytokine production (32). PRRs have been divided into several families: TLRs, RIG-I like receptors, NOD-like receptors (NLRs), C-type lectin receptors, AIM2-like receptors, and cytosol DNA sensors (32). Previous studies have demonstrated that innate immune responses are induced by AAV infection through the TLR9/MyD88 pathway in plasmacytoid DCs (pDCs) (18) and TLR2 in human nonparenchymal liver cells (19). Another study has shown that increased TLR9 signaling was observed in the livers when AAV vectors were administered in mice via systemic administration (30). Zhu et al. found that strong IFN- $\alpha$ secretion was achieved 18 hours after pDCs were infected by AAV transduction (18). The activation of innate immune response was triggered by AAV in nonparenchymal liver cells within 24 hours (19). It has been demonstrated that systemic administration of AAV vectors led to the rapid induction of inflammatory cytokines, which returned to baseline at 6 hours after AAV injection in mice $(30,31)$. This early activation of the innate immune response influences long-term transgene expression after AAV transduction (30). However, the early activation of the innate immune response may not contribute to the declined transgene expression at week 6 in some patients with hemophilia B after AAV administration. In this study, upregulation of IFN- $\beta$ expression was observed at day 6 in HeLa cells following AAV transduction. In primary human hepatocytes, the pattern of IFN- $\beta$ expression was inconsistent. Generally, high expression of IFN- $\beta$ was achieved after day 5 in all samples. Finally, we also detected the upregulation of IFN- $\beta$ in human hepatocytes from humanized mice from week 4 to week 8 after AAV administration. These results strongly support the notion that the activation of innate immune response is elicited from long-term AAV transduction. This may inhibit later transgene expression, as manifested in clinical trials in some patients with hemophilia.

The high expression of IFN- $\beta$ at day 1 of AAV transduction in some primary human hepatocytes may result from the TLR9-mediated innate immune response but not from the TLR2 pathway, as suggested by early studies $(18,19)$. The mechanism of IFN- $\beta$ upregulation at later time points after AAV transduction has not been investigated. It is unlikely that the activation of the innate immune response at the late phase is triggered by the same mechanism as activation at the early phase after AAV transduction. Early after AAV infection, TLR9 recognition of the AAV genome or TLR2 recognition of the AAV capsid plays a major role in pDCs or nonparenchymal liver cells for activation of the innate immune response, respectively $(18,19)$. TLRs only sense PRRs localized on the cell surface or in the endosomes. After longterm AAV transduction, if PRRs from AAV vector (dsDNA AAV genome or AAV capsid protein) still remain in the endosomes, TLRs should continue to recognize these PRRs and induce a sustained IFN- $\beta$ expression. This assumption is contrary to what we observed in this study, since IFN- $\beta$ expression was at baseline levels during days 3-4 after AAV transduction in HeLa cells (Figure 4). Therefore, some other mechanisms may activate the innate immune response at a late phase after AAV transduction. In addition to the transmembrane TLRs, cytoplasmic PRRs can also detect viral nucleic acids or proteins from viral infection. Generally, RIG-I and MDA5 are able to sense cytosolic dsRNA from RNA viruses, and several DNA sensors in cytoplasm have been identified (24). NLR proteins are also involved in the innate immune response to virus infection (32). It has been demonstrated that AAV ITRs have promoter function and the 3'-ITR may transcribe minus-stranded RNA, which serves as antisense RNA that could inhibit transgene production (Supplemental Figure 1). This antisense RNA may bind to sense RNA to form dsRNA via annealing in the cytoplasm. The dsRNA generated from AAV vector transduction has the potential to trigger the dsRNA innate immune response by modulation of RIG-I and MDA5 expression. MDA5 and RIG-I bind to the common adaptor MAVS, which will promote direct or indirect transcriptional induction of many genes via activation of a few essential transcription factors, including interferon-regulatory factors and NF- $\mathrm{kB}$, to produce IFN- $\beta$ and inflammatory cytokines. Indeed, at the late time point after AAV transduction, the activation of MDA5 was observed in HeLa cells, primary human hepatocytes, and hepatocytes from humanized mouse livers. The upregulation of MDA5, but not RIG-I, further supports our hypothesis that dsRNA could be formed from minus-stranded RNA from the AAV 3'-ITR, since MDA5 senses the long dsRNA. This result suggests that dsRNA-mediated activation of the innate immune response is triggered after long-term AAV transduction. Consistently, using siRNA to block the dsRNA sensor, MDA5, or the adaptor, MAVS, IFN- $\beta$ expression was inhibited and transgene expression was increased at the late time points of AAV infection (Figure 9). These results further support that the dsRNA-activated innate immune response contributes to a therapeutic FIX decrease at a later time in 
patients with hemophilia B receiving AAV gene therapy. As to how dsRNA-mediated activation of innate immune response is only detected at a later phase of AAV transduction, one of the possible mechanisms is the length of time needed to generate enough antisense RNA to trigger dsRNA-sensing pathways. The promoter function of the AAV ITR is weak; therefore, it would take a relatively long time to form enough dsRNA to reach a threshold that can be detected by cytoplasmic sensors. In addition, the biology of AAV vector transduction may play a role in dsRNA formation at the late phase of AAV transduction. Unlike adenovirus vectors, the transgene expression reaches its peak at week 6 in preclinical and clinical trials and remains persistent for years after AAV vector administration. Therefore, a high amount minus-stranded RNA can only be synthesized at the late phase of AAV transduction.

Compared with the activation of innate immune responses mediated by dsRNA in HeLa cells, and although IFN- $\beta$ was upregulated in all samples of human primary hepatocytes after AAV transduction, MDA5 upregulation was only observed in primary hepatocytes from 6 of 12 subjects (Figure 6). These differences in the MDA5 regulation may be explained by the different genetic backgrounds that govern the immune system, as shown in patients with hepatitis $\mathrm{C}$ treated with IFN- $\alpha(33,34)$. The difference in MDA upregulation at different time points after AAV transduction in human hepatocytes xenograft mice may be also caused by the varied genetic background of hepatocyte donors. This assumption is supported by the clinical trials in patients with hemophilia B, in which only a subset of patients demonstrated decreased transgene expression at late time points after systemic administration of AAV/FIX vectors, even though the same dose of AAV was used. In future studies, it will be important to identify genetic factors that contribute to the dsRNA-mediated innate immune response after AAV gene therapy. Additionally, the dsRNA-mediated late innate immune response activation from AAV transduction did not occur in every tested cell line, which also highlights that the specific conditions inside of cells play a role in regulating the dsRNA-mediated innate immune response activation. In clinical trials in patients with blindness, some studies reported that the therapeutic effect was progressively improved, reached a peak, and then subsequently decreased in patients with Leber's congenital amaurosis after subretinal administration of AAV vectors encoding therapeutic transgenes $(35,36)$. It was suggested that ongoing retinal degeneration plays a role for the declined sensitivity due to the finding of progressive retinal thinning after AAV gene therapy $(35,37)$. It is unknown whether the innate immunity is activated at the later time points in the eye and thereby influences transgene expression in patients with blindness after AAV gene therapy. Since the number of clinical patients involved in AAV gene therapy studies is still limited, a large number of patients will be needed to address the importance of dsRNA-mediated innate immune responses in different tissues/organs after AAV targeting. Regardless, the results from this study highlight the concern that dsRNA-mediated innate immune response is triggered in human hepatocytes from long-term AAV transduction.

IFN- $\beta$, as a member of the type I IFN family, is one of the downstream effectors in the dsRNA pathway. Type I IFN plays an antiviral role by binding to IFNAR, activating the JAK/STAT pathway, and then inducing a wide array of IFN-stimulated genes (ISGs). ISGs act on different steps of transcription and translation to inhibit protein synthesis (38-41). For example, tripartite motif 22 (TRIM22) inhibits RNA transcription (41), and both ISG15 and ZAP affect eIF4G and eIF4A to inhibit protein synthesis (38). Some ISGs are able to induce cell apoptosis, such as TRAIL and FasL $(42,43)$. Therefore, the multiaspect function of ISGs induced by IFN- $\beta$ contributes to the inhibition of transgene expression.

Although AAV 3'-ITR has weak promoter function, the exact mechanism of dsRNA formation is still unclear. Future studies should focus on elucidation of how dsRNA is formed during long-term AAV transduction. This study also raises another concern, regarding other mechanisms beside cytosolic dsRNA. These other pathways could be triggered by cytosolic dsDNA from the AAV vector genome after AAV vectors escape from the endosomes/lysosomes or from NLR recognition, since it has been previously reported that dsRNA also induces NRPP pathways (44-47). Further studies will be required to address these important issues.

In summary, our study reveals a mechanism through which long-term AAV transduction activates the innate immune response through the cytoplasmic dsRNA recognition pathway, which leads to the production of type I IFN- $\beta$. Transiently blocking the dsRNA pathway decreases IFN- $\beta$ expression and increases transgene expression in AAV-transduced cells. These results provide valuable information that could help the field design effective approaches to blunt the dsRNA response pathways for improvement of AAV transduction in future human clinical trials. 


\section{Methods}

Cells. HeLa cells, HEK293 cells, Huh7 cells, and HepG2 cells were grown in Dulbecco's Modified Eagle's Medium with $10 \%$ FBS and $1 \%$ penicillin-streptomycin at $37^{\circ} \mathrm{C}$ in $5 \% \mathrm{CO}_{2}$.

Human primary hepatocytes were purchased from Triangle Research Labs. Details regarding fresh human primary hepatocytes are listed in Supplemental Table 1. Primary hepatocytes were plated in Williams' E Medium with Hepatocyte Thawing and Plating Supplement Pack (Thermo Fisher Scientific) and maintained in Williams' E Medium with Hepatocyte Maintenance Supplement Pack and HepExtend Supplement (Thermo Fisher Scientific).

$A A V$ virus production. AAV virus production was performed as previously described using the triple plasmid transfection (48). Briefly, HEK293 cells were transfected with an AAV transgene plasmid (sspTR-CBA-luciferase, scpTR-CBh-GFP, sspTR-CMV-GFP, sspTR-CBA-AAT, scpTR-shRNA scramble and scpTR-TTR-FIX-opt), a Rep and Cap AAV helper plasmid, and the adenovirus helper plasmid pXX680. Forty-eight hours after transfection, cells were harvested, lysed, and AAV vectors were purified by cesium chloride $(\mathrm{CsCl})$ gradient density centrifugation. The virus titer was determined by Q-PCR using the vector genome as template and is reported as viral genomes (vg).

Mice. Human xenografted mice with $70 \%$ human hepatocyte repopulation were purchased from Yecuris. Mice were maintained in a specific pathogen-free facility at the University of North Carolina at Chapel Hill.

In vitro transduction. HeLa, Huh7, HEK293, or HepG2 cells were transduced by $5 \times 10^{3}$ vg AAV vectors per cell. Transduced cells were harvested at the indicated time points. For the long-term AAV transduction study, 1 $\times 10^{5} \mathrm{HeLa}$ cells were transduced by $5 \times 10^{3} \mathrm{vg}$ AAV per cell in a 6-well plate. At day 3 after transduction, cells were split 1:5 and then cultured for, at most, 5 days, with the medium changed every day. AAV-transduced cells were harvested at the indicated time points, and cell lysate was used to measure luciferase activity.

Transduction of human primary hepatocytes. Suspended hepatocytes were plated on a collagen I-coated plate, and AAV vectors (AAV2/GFP or AAV2/FIX-opt) were added in a dose of $5 \times 10^{3} \mathrm{vg}$ per cell. One day later, the plating medium was changed to maintenance medium. Primary hepatocytes were cultured for 10 days while the medium was changed every day. Hepatocytes were harvested at different time points for detection of MDA-5, RIG-I, and IFN- $\beta$ mRNA expression.

Mouse experiments. Human hepatocyte xenografted mice were administered $3 \times 10^{11} \mathrm{vg}$ AAV8/FIX-opt via retro-orbital injection. At week 4 or 8 after AAV injection, mice were sacrificed and livers were harvested for RNA extraction and protein analysis via Western blot.

Luciferase assay. Cells transduced by AAV2/luciferase were treated with passive lysis buffer (Promega) for 20 minutes. Luciferase activity was measured with Luciferase Assay Reagent (Promega) following the manufacturer's instructions, using a Victor3 plate reader (Perkin Elmer Inc.)

Transfection assay. For poly (I:C) transfection, cells were transfected with $2 \mu \mathrm{g}$ poly (I:C) using Lipofectamine 3000 (Thermo Fisher Scientific) in a 12-well plate at different time points: 18 hours prior to AAV transduction, at the same time as transduction, or 3 days after AAV transduction. For siRNA transfection, at day 3 after transduction, HeLa cells were split and 24 hours later cells were transfected with $1 \mu \mathrm{g}$ siRNA (siMDA5, CUGAAUCUGCUCCUUCACC; siMAVS, AUACAACUGACCCUGUGGG; siMAVS-2, UAGUUGAUCUCGCGGACGA and CCGUUUGCUGAAGACAAGA; siControl, UGUGAUCAAGGACGCUAUG). At 48 or 72 hours after transfection, cells were harvested for luciferase assay or RNA extraction.

$R N A$ isolation and real-time PCR. RNA from cultured cells or liver tissues was isolated using TRIzol Reagent (Invitrogen). Synthesis of first-strand cDNA from RNA templates was performed using the RevertAid First-Strand cDNA Synthesis Kit (Thermo Fisher Scientific). Real-time PCR was performed using a LightCycler 480 instrument (Roche). Primers used for real-time PCR are listed in Supplemental Table 2.

Western blot. Cells or tissues were treated with RIPA buffer. $60 \mu \mathrm{g}$ total cellular protein per lane was loaded onto an SDS-PAGE gel. The proteins were transferred to a nitrocellulose membrane and stained with rabbit monoclonal MDA5 (700360, Thermo Fisher Scientific) and $\beta$-actin antibody (AM4302, Thermo Fisher Scientific). Signal was detected using ECL Western Blotting Detection Reagent (GE). Data analysis was performed using ImageJ software (NIH).

IFN- $\beta$ promoter reporter assay. $1 \times 10^{5} \mathrm{HeLa}$ cells were transduced by $5 \times 10^{3} \mathrm{vg}$ AAV2/GFP per cell in a 6 -well plate. Cells were split 1:5 at day 3 after transduction. Twenty-four hours later, cells were cotransfected with an IFN- $\beta$ promoter reporter plasmid and siRNA. Then, luciferase activity was measured 72 hours after transfection. 
Minus strand transcription detection. At day 8 after AAV2/luciferase transduction, the HeLa cells were harvested. RNA was isolated using TRIzol Reagent (Invitrogen) and was treated with DNase (Amplification Grade, Thermo Fisher Scientific). Eight pairs of primers for the luciferase gene were used to synthesis plus strand or minus strand cDNA with SuperScript II Reverse Transcriptase (Thermo Fisher Scientific). Two pairs of primers were used for the PCR detection of sense and antisense transcripts. Primers used in this experiment are listed in Supplemental Table 2.

Statistics. All statistical calculations were performed using statistical software (GraphPad Prism 7.0). Data are presented as mean \pm SEM. Data for multiple comparisons were compared using 2-way ANOVA, and data for single comparisons were evaluated by the 2-tailed Student's $t$ test. Differences between different groups were considered to be statistically significant when $P$ values were less than 0.05 .

Study approval. All animal studies were reviewed and approved by the University of North Carolina Institutional Animal Care and Use Committee.

\section{Author contributions}

WS performed experiments, analyzed the data, prepared figures, and wrote the manuscript. LFE, ZC, XC, JS, and TH performed experiments. MD provided reagents. MLH, JT, and RJS analyzed the data and wrote the manuscript. CL conceived and supervised the project, analyzed the data, and wrote the manuscript.

\section{Acknowledgments}

This work was supported by National Institutes of Health grants R01AI117408 (to CL), R01HL125749 (to CL), R01AI072176 and R01AR064369 (to RJS and MLH), and AI109784 and AI029564 (to JT). We would like to thank Amanda Dobbins and Megan Dougherty for their help in the creation of some of the plasmids used in this study.

Address correspondence to: Chengwen Li, Gene Therapy Center, 7007 Thurston-Bowles, CB 7352, University of North Carolina at Chapel Hill, Chapel Hill, North Carolina 27599, USA. Phone: 919.962.7624; Email: chengwen@med.unc.edu.

1. Manno CS, et al. Successful transduction of liver in hemophilia by AAV-factor IX and limitations imposed by the host immune response. Nat Med. 2006;12 (3):342-347.

2. Nathwani AC, et al. Adenovirus-associated virus vector-mediated gene transfer in hemophilia B. N Engl J Med. 2011;365 (25):2357-2365

3. Nathwani AC, et al. Long-term safety and efficacy of factor IX gene therapy in hemophilia B. N Engl J Med. 2014;371 (21):1994-2004.

4. Nathwani AC, Davidoff AM, Tuddenham EGD. Advances in gene therapy for hemophilia. Hum Gene Ther. 2017;28(11):1004-1012.

5. Wu Z, et al. Optimization of self-complementary AAV vectors for liver-directed expression results in sustained correction of hemophilia B at low vector dose. Mol Ther. 2008;16 (2):280-289.

6. Monahan PE, et al. Employing a gain-of-function factor IX variant R338L to advance the efficacy and safety of hemophilia B human gene therapy: preclinical evaluation supporting an ongoing adeno-associated virus clinical trial. Hum Gene Ther. 2015;26 (2):69-81.

7. McIntosh J, et al. Therapeutic levels of FVIII following a single peripheral vein administration of rAAV vector encoding a novel human factor VIII variant. Blood. 2013;121 (17):3335-3344

8. Nathwani AC, et al. Long-term safety and efficacy following systemic administration of a self-complementary AAV vector encoding human FIX pseudotyped with serotype 5 and 8 capsid proteins. Mol Ther. 2011;19 (5):876-885.

9. Nathwani AC, et al. Self-complementary adeno-associated virus vectors containing a novel liver-specific human factor IX expression cassette enable highly efficient transduction of murine and nonhuman primate liver. Blood. 2006;107 (7):2653-2661.

10. Nathwani AC, et al. Safe and efficient transduction of the liver after peripheral vein infusion of self-complementary AAV vector results in stable therapeutic expression of human FIX in nonhuman primates. Blood. 2007;109 (4):1414-1421.

11. Sabatino DE, et al. Efficacy and safety of long-term prophylaxis in severe hemophilia A dogs following liver gene therapy using AAV vectors. Mol Ther. 2011;19 (3):442-449.

12. Sarkar R, et al. Long-term efficacy of adeno-associated virus serotypes 8 and 9 in hemophilia a dogs and mice. Hum Gene Ther 2006;17 (4):427-439.

13. Wang L, et al. Sustained correction of disease in naive and AAV2-pretreated hemophilia B dogs: AAV2/8-mediated, liver-directed gene therapy. Blood. 2005;105 (8):3079-3086

14. Pien GC, et al. Capsid antigen presentation flags human hepatocytes for destruction after transduction by adeno-associated viral vectors. J Clin Invest. 2009;119 (6):1688-1695.

15. Li C, et al. Cellular immune response to cryptic epitopes during therapeutic gene transfer. Proc Natl Acad Sci USA. 2009;106 (26):10770-10774 
16. Wu TL, et al. CD8+ T cell recognition of epitopes within the capsid of adeno-associated virus 8-based gene transfer vectors depends on vectors' genome. Mol Ther. 2014;22(1):42-51.

17. He Y, et al. Kinetics of adeno-associated virus serotype 2 (AAV2) and AAV8 capsid antigen presentation in vivo are identical. Hum Gene Ther. 2013;24 (5):545-553.

18. Zhu J, Huang X, Yang Y. The TLR9-MyD88 pathway is critical for adaptive immune responses to adeno-associated virus gene therapy vectors in mice. J Clin Invest. 2009;119 (8):2388-2398.

19. Hösel M, et al. Toll-like receptor 2-mediated innate immune response in human nonparenchymal liver cells toward adeno-associated viral vectors. Hepatology. 2012;55 (1):287-297.

20. Flotte TR, et al. Expression of the cystic fibrosis transmembrane conductance regulator from a novel adeno-associated virus promoter. J Biol Chem. 1993;268 (5):3781-3790.

21. Haberman RP, McCown TJ, Samulski RJ. Novel transcriptional regulatory signals in the adeno-associated virus terminal repeat A/D junction element. J Virol. 2000;74 (18):8732-8739.

22. Wei W, Pelechano V, Järvelin AI, Steinmetz LM. Functional consequences of bidirectional promoters. Trends Genet. 2011;27 (7):267-276

23. Schlee M. Master sensors of pathogenic RNA - RIG-I like receptors. Immunobiology. 2013;218 (11):1322-1335.

24. Schlee M, Hartmann G. Discriminating self from non-self in nucleic acid sensing. Nat Rev Immunol. 2016;16 (9):566-580.

25. Kell AM, Gale M. RIG-I in RNA virus recognition. Virology. 2015;479-480:110-121

26. Kato $\mathrm{H}$, et al. Length-dependent recognition of double-stranded ribonucleic acids by retinoic acid-inducible gene-I and melanoma differentiation-associated gene 5. J Exp Med. 2008;205 (7):1601-1610.

27. Reikine S, Nguyen JB, Modis Y. Pattern recognition and signaling mechanisms of RIG-I and MDA5. Front Immunol. 2014;5:342.

28. Triantafilou K, Vakakis E, Kar S, Richer E, Evans GL, Triantafilou M. Visualisation of direct interaction of MDA5 and the dsRNA replicative intermediate form of positive strand RNA viruses. J Cell Sci. 2012;125 (Pt 20):4761-4769.

29. Kato H, et al. Differential roles of MDA5 and RIG-I helicases in the recognition of RNA viruses. Nature. 2006 ;441 (7089):101-105.

30. Martino AT, et al. The genome of self-complementary adeno-associated viral vectors increases Toll-like receptor 9-dependent innate immune responses in the liver. Blood. 2011;117 (24):6459-6468.

31. Zaiss AK, Liu Q, Bowen GP, Wong NC, Bartlett JS, Muruve DA. Differential activation of innate immune responses by adenovirus and adeno-associated virus vectors. J Virol. 2002;76 (9):4580-4590.

32. Brubaker SW, Bonham KS, Zanoni I, Kagan JC. Innate immune pattern recognition: a cell biological perspective. Annu Rev Immunol. 2015;33:257-290.

33. Tavis JE, Donlin MJ, Aurora R, Fan X, Di Bisceglie AM. Prospects for personalizing antiviral therapy for hepatitis C virus with pharmacogenetics. Genome Med. 2011;3 (2):8

34. Sheppard P, et al. IL-28, IL-29 and their class II cytokine receptor IL-28R. Nat Immunol. 2003;4 (1):63-68

35. Bainbridge JW, et al. Long-term effect of gene therapy on Leber's congenital amaurosis. N Engl J Med. 2015;372(20):1887-1897.

36. Jacobson SG, et al. Improvement and decline in vision with gene therapy in childhood blindness. N Engl J Med. 2015;372 (20):1920-1926

37. Cideciyan AV, et al. Human retinal gene therapy for Leber congenital amaurosis shows advancing retinal degeneration despite enduring visual improvement. Proc Natl Acad Sci USA. 2013;110 (6):E517-E525.

38. Li MM, MacDonald MR, Rice CM. To translate, or not to translate: viral and host mRNA regulation by interferon-stimulated genes. Trends Cell Biol. 2015;25(6):320-329.

39. Schoggins JW, Rice CM. Interferon-stimulated genes and their antiviral effector functions. Curr Opin Virol. 2011;1 (6):519-525.

40. Diamond MS, Farzan M. The broad-spectrum antiviral functions of IFIT and IFITM proteins. Nat Rev Immunol. 2013;13 (1):46-57.

41. Schneider WM, Chevillotte MD, Rice CM. Interferon-stimulated genes: a complex web of host defenses. Annu Rev Immunol. 2014;32:513-545.

42. Chawla-Sarkar M, et al. Apoptosis and interferons: role of interferon-stimulated genes as mediators of apoptosis. Apoptosis. 2003;8 (3):237-249

43. Kotredes KP, Gamero AM. Interferons as inducers of apoptosis in malignant cells. J Interferon Cytokine Res. 2013;33 (4):162-170

44. Allen IC, et al. The NLRP3 inflammasome mediates in vivo innate immunity to influenza A virus through recognition of viral RNA. Immunity. 2009;30 (4):556-565.

45. Kanneganti TD, et al. Critical role for Cryopyrin/Nalp3 in activation of caspase-1 in response to viral infection and double-stranded RNA. J Biol Chem. 2006;281 (48):36560-36568.

46. Sabbah A, et al. Activation of innate immune antiviral responses by Nod2. Nat Immunol. 2009;10 (10):1073-1080.

47. Kapoor A, Forman M, Arav-Boger R. Activation of nucleotide oligomerization domain 2 (NOD2) by human cytomegalovirus initiates innate immune responses and restricts virus replication. PLOS ONE. 2014;9 (3):e92704

48. Xiao X, Li J, Samulski RJ. Production of high-titer recombinant adeno-associated virus vectors in the absence of helper adenovirus. J Virol. 1998;72 (3):2224-2232. 